\title{
Anti-CD22-CAR m971-BBz Lentiviral Vector-transduced Autologous T Lymphocytes
}

National Cancer Institute

\section{Source}

National Cancer Institute. Anti-CD22-CAR m971-BBz Lentiviral Vector-transduced

Autologous T Lymphocytes. NCI Thesaurus. Code C120035.

Autologous human T-lymphocytes transduced with a recombinant lentiviral vector encoding a chimeric T-cell receptor (chimeric antigen receptor or CAR) consisting of an anti-CD22 sing le chain variable fragment (scFv) derived from the monoclonal antibody (moAb) 971 (m971), and the co-stimulatory domain 4-1BB (CD137) coupled to the zeta chain of the TCR/CD3 complex (CD3-zeta), with potential immunostimulating and antineoplastic activities. Autologous peripheral blood lymphocytes (PBLs) from a patient with CD22-positive cancer are transduced with this lentiviral vector that encodes the CAR gene specific for CD22. After expansion in culture and reintroduction into the patient, the anti-CD22-CAR m971-BBz lentiviral vector-transduced autologous T-lymphocytes express anti-CD22-CAR on their cell surfaces and bind to the CD22 antigen on tumor cell surfaces. Subsequently, CD22-expressing tumor cells are lysed. CD22, a B-lineagerestricted, transmembrane phosphoglycoprotein, is expressed on malignant B-cells. m971 binds to a membrane proximal epitope on CD22 and has a higher binding affinity compared to other anti-CD22 moAb. 